\title{
Análisis de la calidad ambiental en un sector del Río Chico (Santa Cruz, Argentina) basado en bioindicadores bentónicos
}

\section{Environmental quality analysis of a sector of the Chico river (Santa Cruz, Argentina) based on benthic indicators}

\author{
Santiago TORRES $S^{1,2 \text { y } 3}$, santiagohtorres@outlook.com \\ Juan Pablo MARTIN ${ }^{13}$, Lucia GÁRGANO ${ }^{2 \text { y } 3}$, Laura ARMENDÁRIZ ${ }^{4} 5$ \\ ${ }^{1}$ Instituto de Ciencias del Ambiente, Sustentabilidad y Ciencias Naturales (ICASUR), Unidad \\ Académica San Julián, Universidad Nacional de la Patagonia. \\ ${ }^{2}$ Consejo Nacional de Investigaciones Científicas y Técnicas (CONICET). \\ ${ }^{3}$ Centro de Investigaciones y Transferencia Santa Cruz (CONICET-UNPA-UTN). \\ ${ }^{4}$ Instituto de Limnología Dr. Raul Ringuelet (UNLP-CONICET) \\ ${ }^{5}$ Facultad de Ciencias Naturales y Museo (FCNyM-UNLP)
}

Recibido: 26/11/2018. Aceptado: 03/04/2019.

\section{RESUMEN}

El río Chico, ubicado en el centro de Santa Cruz, recorre $600 \mathrm{~km}$ desde sus nacientes hasta su desembocadura. En su tramo medio, se encuentra la localidad de Gobernador Gregores $\left(48^{\circ} 45^{\prime} 3^{\prime \prime S}, 70^{\circ} 14^{\prime} 54^{\prime \prime} \mathrm{O}\right)$, que ejerce su influencia sobre el río a través de las actividades agropecuarias y el vertido de efluentes urbanos. Con el objetivo de conocer el grado de impacto sobre la calidad del agua del río, se realizó un análisis de la comunidad de macroinvertebrados bentónicos y se calculó el índice Biotic Monitoring Patagonian Streams (BMPS). En marzo de 2017, se efectuó un primer relevamiento en seis estaciones de muestreo dispuestas en un diseño de gradiente de impacto, con dos estaciones control aguas arriba de la localidad y cuatro estaciones aguas abajo. En cada estación se tomaron muestras de agua para análisis fisicoquímicos, se registraron datos ambientales mediante sonda multiparamétrica y se recolectaron cuatro muestras de macrobentos utilizando una red Surber con malla de 0,5 $\mathrm{mm}$ de abertura. Los organismos recolectados fueron identificados y contabilizados bajo microscopios estereoscópico y óptico. Para cada muestra se calculó el número de taxones (S), la abundancia total $(\mathrm{N})$, la diversidad $\left(\mathrm{H}^{\prime}\right)$ y la equitatividad $(\mathrm{J})$; los datos de abundancia se analizaron utilizando estadística multivariada. Se identificaron 25 taxones, siendo los mejor representados los oligoquetos Naididae, los hirudíneos, los moluscos Chilinidae y Lymnaeidae, y los insectos Chironomidae, Baetidae y Glossosomatidae. En las estaciones ubicadas aguas abajo más cercanas a la localidad, la comunidad se encontró dominada ampliamente por oligoquetos y quironómidos, y presentó los valores más bajos de S, H' y J. Los valores de BMPS en esas estaciones indicaron la existencia de aguas contaminadas a fuertemente contaminadas. En las estaciones control y las más alejadas aguas abajo, los valores de $\mathrm{S}, \mathrm{H}^{\prime}$ y $\mathrm{J}$ aumentaron significativamente y el índice BMPS indicó mejores condiciones en la calidad ambiental del río.

Palabras clave: Bentos; Bioindicadores; Macroinvertebrados; Calidad ambiental; Patagonia austral. 


\begin{abstract}
The Chico river, located in the center of Santa Cruz, runs $600 \mathrm{~km}$ from its source to its mouth. In its middle reaches, is the town of Gobernador Gregores ( $\left.48^{\circ} 45^{\prime} 33^{\prime \prime} \mathrm{S}, 70^{\circ} 14^{\prime} 54^{\prime \prime} \mathrm{W}\right)$, which exerts influence on the river through agricultural activities and the discharge of urban sewages. In order to know the degree of impact on the water quality of the river, the analysis of the community of benthic macroinvertebrates was carried out and the Biotic Monitoring Patagonian Streams (BMPS) index was calculated. A first survey was performed in March 2017 at six sampling stations arranged in a gradient design, with two control stations upstream and four stations downstream of the town. At each station, water samples were taken for physicochemical analysis, environmental data were recorded with a multiparameter probe, and four samples of macrobenthos were collected using a Surber network with $0.5 \mathrm{~mm}$ mesh. The collected organisms were identified and counted under optic and stereoscopic microscopes. For each sample, species richness $(\mathrm{S})$, total abundance $(\mathrm{N})$, diversity $\left(\mathrm{H}^{\prime}\right)$ and evenness $(\mathrm{J})$ were calculated, and the abundance data were analyzed using multivariate statistics. A total of 25 taxa were identified. The best represented were the oligochaetes Naididae, the hirudineans, the mollusks Chilinidae and Lymnaeidae, and the insects Chironomidae, Baetidae and Glossosomatidae. At the stations located downstream closest to the town the community was dominated by oligochaetes and chironomids, and presented the lowest values of S, H' and J. The values of BMPS at these stations indicated the existence of polluted to heavily polluted water. At the control and the furthest downstream stations, the values of S, H' and J increased significantly, and the BMPS index indicated better conditions in the environmental quality of the river.
\end{abstract}

Key words: Benthos; Bioindicators; Macroinvertebrates; Environmental quality; Southern Patagonia.

\title{
1. INTRODUCCIÓN
}

En las últimas décadas, los sistemas fluviales han sido sometidos a fuertes presiones ambientales. La modificación de las cuencas hidrográficas, los cambios de uso de la tierra, la sobreexplotación de aguas para actividades humanas y la contaminación de los recursos acuáticos, impactan en los ambientes lóticos alterando las propiedades físicas, la composición química y la estructura de su biocenosis (Miserendino, 2004; Masi y Miserendino, 2009; Asueta et al., 2011; Armendariz et al., 2017).

Por su alta sensibilidad a los cambios ambientales, las comunidades presentes en los ecosistemas acuáticos, en particular la del macrobentos, han sido tradicionalmente utilizadas para el monitoreo de los cambios en la calidad del agua en ríos y arroyos. Numerosos trabajos, realizados en diferentes regiones geográficas, han demostrado que las comunidades bentónicas tienen la capacidad de reflejar las anomalías y procesos de degradación tan frecuentes en los ambientes acuáticos (Weatherley y Ormerod, 1992; Rosenberg y Resh, 1993; Suren 2000; Roy et al., 2003). Los cambios que se producen en la abundancia y la dominancia de taxones en la comunidad bentónica en respuesta a un disturbio, dependerán del nivel de alteración de las condiciones ambientales y del grado de sensibilidad o tolerancia de las especies que integran la comunidad (Martin et al., 2014). Las alteraciones ambientales de alto o mediano impacto pueden generar cambios importantes en la estructura de la comunidad, como consecuencia de la desaparición de especies sensibles, el aumento en la abundancia de especies tolerantes o la aparición de nuevas especies (Prat et al., 2009). Existen 
además, alteraciones ambientales que no se verían reflejadas en la estructura comunitaria, pero que tienen su efecto a nivel individual, como es el caso de la expresión del mecanismo de defensa multixenobiotico estudiado en muchos invertebrados acuáticos (Prat et al., 2009; Assef et al., 2014; Horak et al., 2017).

El estudio de las respuestas de las comunidades bentónicas frente a disturbios ambientales han conducido al diseño de distintos índices bióticos que permiten ponderar dichas alteraciones (Prat et al., 2009). Entre los más utilizados se encuentran el Biological Monitoring Working Party (BMWP) y el Average Score Per Taxon (ASPT), los cuales combinan el número de taxa totales (a nivel de Familia) con un valor conocido de tolerancia y sensibilidad a la contaminación orgánica (Prat et al., 2009); o el EPT (Ephemeroptera, Plecoptera y Trichoptera) que se basa en la abundancia relativa de los tres órdenes de insectos más sensibles a la contaminación (Gray y Delaney, 2008). En Argentina, muchos de estos índices han sido adaptados para su aplicación en distintas ecorregiones del país (Prat et al., 2009). El Biotic Index for Pampean rivers and streams (IBPAMP) o el Biotic Monitoring Patagonian Streams (BMPS) son adaptaciones del índice BMWP a la región pampeana y a la región andino-patagónica respectivamente, y han sido utilizados para el diagnóstico de la calidad ambiental en el medio acuático (Miserendino y Pizzolón, 1999, Rodrigues Capítulo et al., 2001, Armendáriz et al., 2017). La adaptación de estos índices se basó, en parte, en la información ecológica de las especies autóctonas, aunque en muchos casos, cuando la información regional no estuvo disponible, se adoptó el grado de sensibilidad o tolerancia determinado a nivel de familia para otras regiones del mundo (Prat et al., 2009).

Entre las principales problemáticas ambientales que enfrenta la provincia de Santa Cruz se pueden mencionar la actividad minera, la ganadería extensiva de ganado ovino y bovino, la falta de planificación en el desarrollo urbano, el vertido de efluentes urbanos e industriales al medio y las represas hidroeléctricas actualmente en construcción sobre el río Santa Cruz (Martin et al., 2014; Tagliaferro et al., 2013; Tagliaferro et al., 2014). Estas actividades afectan de manera negativa a los cuerpos de agua dulce de la provincia, recurso de por sí escaso en la región. Estudios integrados, en diversos ambientes y con métricas claras para la comparación del estado de los ambientes acuáticos continentales, son fundamentales para llevar adelante estrategias de manejo y la implementación de medidas de mitigación ante el potencial impacto causado por las actividades antrópicas.

El río Chico, ubicado en el centro de Santa Cruz, recorre $600 \mathrm{~km}$ desde sus nacientes, en la Sierra de las Vacas, hasta su desembocadura (Figura 1). Los principales tributarios son el río Chalía y el río Belgrano, llegando a un caudal máximo de $31,340 \mathrm{~m}^{3} \mathrm{~s}^{-1}$ en primavera (Red Hidrológica Nacional, 2013). En el curso medio del río, en el departamento Río Chico, se encuentra la localidad de Gobernador Gregores $\left(48^{\circ} 46^{\prime} \mathrm{S}, 70^{\circ} 15^{\prime} \mathrm{O}\right)$, cuyas principales actividades económicas son la ganadería extensiva de bovinos y la agricultura a pequeña y mediana escala (Godoy Manriquez, 2000). Los efluentes urbanos generados en la localidad de Gobernador Gregores son vertidos en el brazo norte del río Chico, previo paso por la planta de tratamiento. Este aporte, sumado al proveniente de la actividad agropecuaria, le proporciona al río un agregado de nutrientes y materia orgánica que podrían generar problemas de eutrofización y enriquecimiento orgánico, ocasionando importantes alteraciones en el ambiente fluvial.

Los objetivos del presente trabajo fueron: (1) analizar el efecto del aporte de la actividad antrópica sobre la calidad ambiental de un sector del río Chico, evaluando los cambios en la estructura y composición de la comunidad bentónica; (2) evaluar la respuesta del índice Biotic 
Monitoring Patagonian Streams (BMPS) (Miserendino y Pizzolón, 1999) ante las alteraciones ambientales observadas en el área de estudio. Los resultados obtenidos nos permitirán ajustar y realizar adaptaciones del índice BMPS para su aplicación en otros cuerpos de agua dulce de la región.

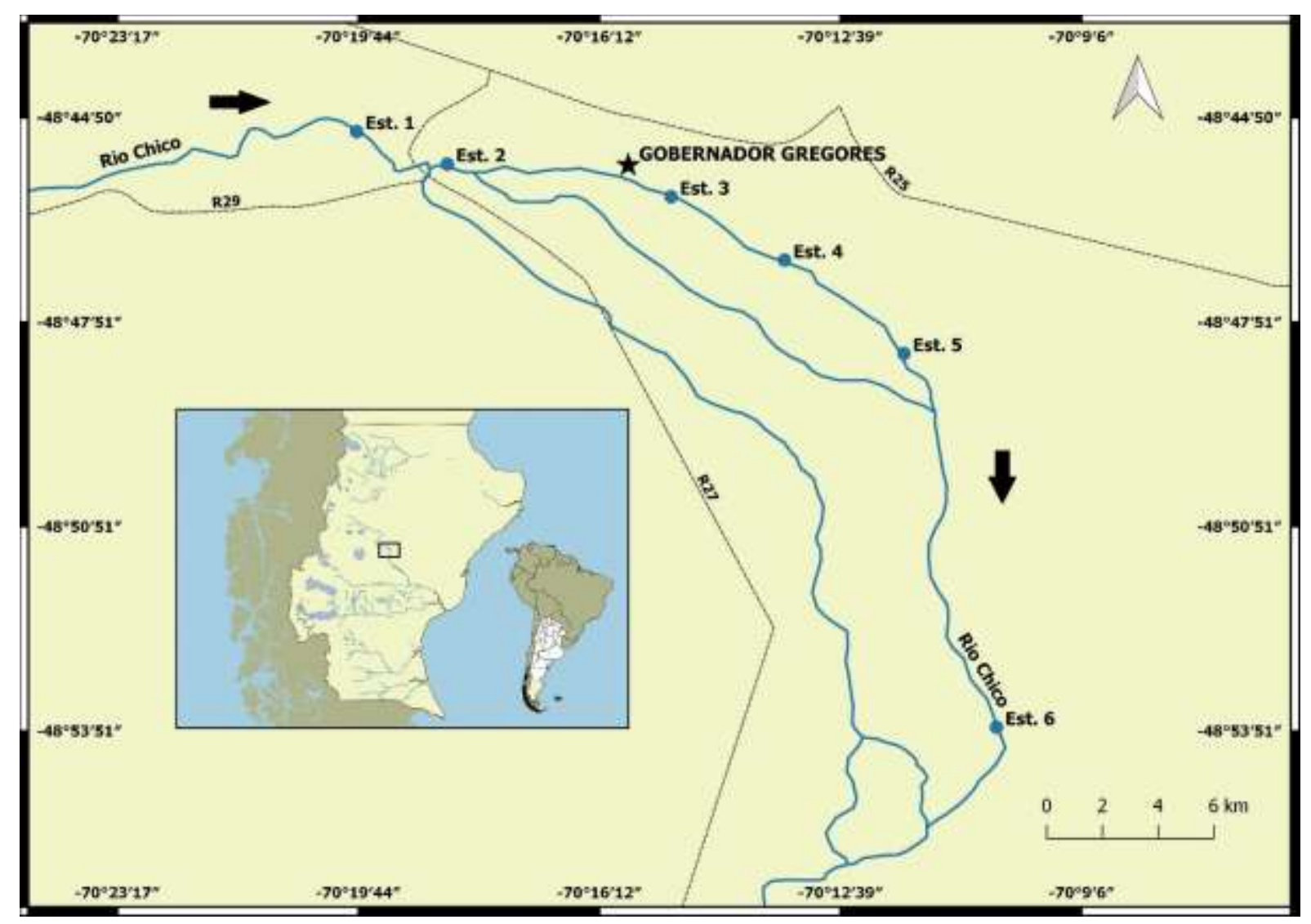

Figura 1. Área de estudio y ubicación de las estaciones de muestreo en el río Chico.

\section{MATERIALES Y METODOS}

En marzo de 2017 se efectuó un primer relevamiento en seis estaciones de muestreo dispuestas en un diseño de gradiente de impacto, con dos estaciones control aguas arriba de la localidad (estaciones 1 y 2) y cuatro estaciones aguas abajo (estaciones 3, 4, 5 y 6) (Figura 1). En cada estación se tomaron in situ datos de $\mathrm{pH}$, temperatura, conductividad, turbidez y concentración de oxígeno disuelto en el agua mediante una sonda multiparamétrica Horiba U10, se midió el ancho del cauce, se registró la velocidad de la corriente con un correntómetro Tecmes TS 1001 y se colectaron muestras de agua para análisis fisicoquímicos en laboratorio. En cada relevamiento se tomaron cuatro muestras de la comunidad de macroinvertebrados bentónicos por estación, utilizando una red manual tipo Surber con boca de 30 x $50 \mathrm{~cm}$ y malla de $500 \mu \mathrm{m}$, y un marco metálico para delimitar un área de muestreo de $0,25 \mathrm{~m}^{2}$ sobre el fondo (Martin et al., 2014, Rivera Velasco et al., 2017). Las réplicas se tomaron en una transecta paralela a la margen del curso de agua en ambientes de correderas con lecho de grava, a una profundidad de entre 30 y $50 \mathrm{~cm}$. El sustrato fue removido en forma manual durante 2 minutos. Las muestras se fijaron in situ con solución de formol al 5\% y posteriormente se guardaron en alcohol al 70\% para su preservación (Martin et al., 2014). Para cada muestra se calculó la diversidad (H') utilizando el índice de Shannon-Wiener, la 
equitatividad (J) utilizando el índice de Pielou, la riqueza específica (S) y la abundancia total $(\mathrm{N})$. Los valores de estos parámetros fueron comparados entre estaciones de muestreo mediante la prueba no paramétrica de Kruskal-Wallis. Las comparaciones post-hoc entre pares de estaciones se realizaron con la prueba no paramétrica de Mann-Whitney. Los datos de abundancia fueron analizados mediante estadística multivariada, utilizando técnicas de agrupamiento (Hierarchical Agglomerative Clustering, HAC) y de ordenamiento no paramétrico (Multidimensional Scaling, MDS), por medio del software PRIMER versión 6.1. Los datos fueron previamente transformados utilizando raíz cuadrada y posteriormente se aplicó el índice de similitud de Bray-Curtis. Para poner a prueba la hipótesis de diferencias en la composición de la comunidad entre estaciones de muestreo se utilizó el test de permutación ANOSIM. La significancia estadística de los agrupamientos entre muestras fue corroborada mediante el análisis de similitud SIMPROF y para determinar la contribución de las especies a la similitud entre muestras y a la disimilitud entre los diferentes grupos de muestras se aplicó la rutina SIMPER (Clarke y Warwick, 2001). El índice Biotic Monitoring Patagonian Streams (BMPS) fue calculado para cada muestra según Miserendino y Pizzolón (1999) y los valores obtenidos en las distintas estaciones de muestreo fueron comparados estadísticamente mediante la prueba de Kruskal-Wallis. Las comparaciones post-hoc entre pares de estaciones se realizaron con la prueba de Mann-Whitney.

\section{RESULTADOS}

Las tablas 1 y 2 muestran los valores obtenidos de las variables ambientales medidas in situ y en laboratorio respectivamente. Los valores de fósforo total (P Total) y amonio $\left(\mathrm{N}-\mathrm{NH}_{4}\right)$ en agua alcanzaron su valor más alto en las estaciones 3 y 4 , al igual que el contenido de materia orgánica en suspensión y el porcentaje de materia orgánica en sólidos en suspensión en agua.

Tabla 1. Datos ambientales tomados in situ.

\begin{tabular}{cccccccc}
\hline Estación & $\mathbf{p H}$ & $\begin{array}{c}\text { Conductividad } \\
\mathbf{m S ~ c m}^{-1}\end{array}$ & $\begin{array}{c}\text { Turbidez } \\
\text { UNT }\end{array}$ & $\begin{array}{r}\text { Ox. Dis. } \\
\mathbf{m g ~ I}^{\mathbf{1}}\end{array}$ & $\begin{array}{r}\text { Temp. } \\
{ }^{\circ} \mathbf{C}\end{array}$ & $\begin{array}{c}\text { Velocidad } \\
\mathbf{m ~ s}^{-1}\end{array}$ & $\begin{array}{c}\text { Ancho } \\
\text { cauce }\end{array}$ \\
\hline 1 & 8,36 & 0,11 & 180,00 & 10,31 & 12,33 & 0,53 & 33,00 \\
\hline 2 & 8,46 & 0,11 & 200,00 & 9,96 & 13,00 & 0,41 & 10,00 \\
3 & 7,93 & 0,15 & 193,33 & 9,30 & 10,00 & 0,22 & 11,00 \\
4 & 7,40 & 0,15 & 213,33 & 8,41 & 13,00 & 0,35 & 9,50 \\
5 & 7,90 & 0,12 & 176,66 & 9,68 & 11,00 & 0,32 & 25,00 \\
6 & 7,66 & 0,12 & 166,66 & 9,68 & 11,00 & 0,72 & 53,00 \\
\hline
\end{tabular}


Tabla 2. Datos ambientales de análisis de laboratorio.

\begin{tabular}{|c|c|c|c|c|c|c|c|c|c|c|}
\hline $\begin{array}{c}\text { Estació } \\
\mathbf{n}\end{array}$ & $\begin{array}{l}\text { Alcalinidad } \\
\underset{1}{\mathrm{mg} \mathrm{CaCO}_{3} \mathrm{I}^{-}}\end{array}$ & $\begin{array}{l}\text { Calcio } \\
\mathrm{mg} \mathrm{l}^{-1} \\
\end{array}$ & $\begin{array}{c}\text { P } \\
\text { TOTAL } \\
\text { mg l}^{-1}\end{array}$ & $\begin{array}{l}\text { P-PO4 } \\
\text { mg l }^{-1} \\
\end{array}$ & $\begin{array}{c}\mathrm{N}- \\
\mathrm{NO}_{3} \\
\mathrm{mg} \mathrm{l}^{-1}\end{array}$ & $\begin{array}{c}\mathrm{N}- \\
\mathrm{NO}_{2} \\
\mathrm{mg} \mathrm{l}^{-1}\end{array}$ & $\begin{array}{l}\mathrm{N}-\mathrm{NH}_{4} \\
\mathrm{mg} \mathrm{l}^{-1} \\
\end{array}$ & $\begin{array}{c}\text { TDS } \\
\text { mg l}^{-1} \\
\end{array}$ & $\begin{array}{c}\text { MO en } \\
\text { sól. en } \\
\text { susp. } \\
\\
\% \\
\end{array}$ & $\begin{array}{c}\text { MO } \\
\text { en } \\
\text { susp. }\end{array}$ \\
\hline 1 & 48,250 & 23,580 & 0,076 & 0,009 & 0,111 & 0,002 & 0,074 & 49,200 & 11,788 & 5,800 \\
\hline 2 & 47,000 & 27,370 & 0,071 & 0,026 & 0,105 & 0,002 & 0,002 & 46,800 & 9,402 & 4,400 \\
\hline 3 & 49,550 & 31,160 & 0,194 & 0,126 & 0,118 & 0,004 & 1,077 & 33,400 & 19,162 & 6,400 \\
\hline 4 & 62,250 & 26,530 & 0,149 & 0,040 & 0,381 & 0,015 & 0,344 & 48,600 & 15,226 & 7,400 \\
\hline 5 & 55,900 & 31,290 & 0,078 & 0,012 & 0,119 & 0,002 & $<0,001$ & 38,600 & 12,953 & 5,000 \\
\hline 6 & 51,000 & 25,680 & 0,065 & 0,011 & 0,123 & 0,001 & $<0,001$ & 43,200 & 11,111 & 4,800 \\
\hline
\end{tabular}

Se identificaron 25 taxones en las muestras, siendo los mejor representados en términos de abundancia los anélidos oligoquetos Naididae, Limnodrilus hoffmeisteri y Nais variabilis, e hirudíneos del género Helobdella sp., los moluscos gasterópodos Chilinidae y Lymnaeidae, e insectos de las familias Chironomidae, Baetidae y Glossosomatidae. El análisis estadístico mostró diferencias significativas en los valores de diversidad ( $\left.\mathrm{H}^{\prime}\right)(\mathrm{H}=18,88, \mathrm{p}=0,002)$, de equitatividad $(\mathrm{J})(\mathrm{H}=19,77, \mathrm{p}=0,001)$, de riqueza específica $(\mathrm{S})(\mathrm{H}=12,80, \mathrm{p}=0,022)$ y de abundancia total $(\mathrm{N})(\mathrm{H}=20,45, \mathrm{p}=0,001)$ entre las estaciones de muestreo. En las estaciones más cercanas al sitio de vertido de efluentes (Est. 3 y 4), la comunidad del macrobentos se encontró ampliamente dominada por oligoquetos Naididae e insectos Chironomidae (Tabla 3), y presentó los valores más altos de $\mathrm{N}$ y los más bajos de H', J y S (Fig. 2). En las estaciones control (estaciones 1 y 2) y las más alejadas aguas abajo (estaciones 5 y 6 ) adquirieron mayor importancia los moluscos Chilina sp. y Lymnaeidae, junto a los insectos de las familias Baetidae, Glossosomatidae y Gripopterygidae (Tabla 3); los valores de H', J y S aumentaron significativamente (Fig. 2). 
Tabla 3. Abundancia promedio ( \pm d.s.) de los diferentes taxones en la comunidad bentónica.

\begin{tabular}{|c|c|c|c|c|c|c|}
\hline & Estación 1 & Estación 2 & Estación 3 & Estación 4 & Estación 5 & Estación 6 \\
\hline Chilinidae & $\begin{array}{c}98,25( \pm \\
16,6)\end{array}$ & 0 & $0,5( \pm 1)$ & 0 & $\begin{array}{c}70,25( \pm \\
24,27)\end{array}$ & $3( \pm 2,16)$ \\
\hline Lymnaeidae & $\begin{array}{c}0,75( \pm \\
1,5)\end{array}$ & $\begin{array}{c}104,5( \pm \\
19,76)\end{array}$ & $0,5( \pm 1)$ & $0,25( \pm 0,5)$ & $0,5( \pm 1)$ & $0,75( \pm 1,5)$ \\
\hline Cochliopidae & 0 & 0 & $5,5( \pm 6,03)$ & 0 & $0,5( \pm 0,58)$ & 0 \\
\hline Sphaeriidae & 0 & 0 & $1( \pm 1,15)$ & $7( \pm 8,29)$ & 0 & 0 \\
\hline Chironomidae & $87( \pm 170)$ & $3,5( \pm 2,38)$ & $\begin{array}{c}773,25( \pm \\
125,96)\end{array}$ & $1210( \pm 140,95)$ & $3( \pm 1,41)$ & $\begin{array}{c}2,75( \pm \\
2,06)\end{array}$ \\
\hline Simulidae & $4( \pm 2,45)$ & $1,5( \pm 1,29)$ & 0 & 0 & $1,75( \pm 0,96)$ & $4,5( \pm 2,38)$ \\
\hline Empididae & $1( \pm 0,82)$ & $2( \pm 0,82)$ & 0 & 0 & $1,75( \pm 1,26)$ & $0,25( \pm 0,5)$ \\
\hline Elmidae & $\begin{array}{c}11( \pm \\
16,71)\end{array}$ & $15,5( \pm 9,4)$ & 0 & 0 & $2( \pm 1,41)$ & $\begin{array}{c}1,25( \pm \\
0,96)\end{array}$ \\
\hline Baetidae & $\begin{array}{c}22,5( \pm \\
11,24)\end{array}$ & $\begin{array}{c}35,5( \pm \\
17,46)\end{array}$ & $0,5( \pm 1)$ & $0,5( \pm 1)$ & $18,75( \pm 6,4)$ & $\begin{array}{c}44,25( \pm \\
4,43)\end{array}$ \\
\hline Nesameletidae & $\begin{array}{c}0,5( \pm \\
0,58)\end{array}$ & $0,5( \pm 1)$ & 0 & 0 & 0 & 0 \\
\hline Glossosomatidae & $0,25( \pm 0,5)$ & 0 & 0 & 0 & $15,5( \pm 8,81)$ & $5( \pm 5,03)$ \\
\hline Hydroptilidae & $\begin{array}{c}0,25( \pm \\
0,5)\end{array}$ & $1( \pm 1,41)$ & $0,25( \pm 0,5)$ & 0 & $2( \pm 1,83)$ & $0,25( \pm 0,5)$ \\
\hline Hydropsychidae & $\begin{array}{c}1,5( \pm \\
1,29)\end{array}$ & $3,25( \pm 2,99)$ & 0 & 0 & $1,75( \pm 0,96)$ & $\begin{array}{c}3,75( \pm \\
2,63)\end{array}$ \\
\hline Hydrobiosidae & $\begin{array}{c}1,5( \pm \\
1,29)\end{array}$ & $1,5( \pm 1)$ & 0 & 0 & $0,25(0,5)$ & $3,5( \pm 1)$ \\
\hline Hemiptera & $0,5( \pm 1)$ & 0 & $2,5( \pm 3,79)$ & $3( \pm 4,76)$ & 0 & 0 \\
\hline Helobdella sp. & $\begin{array}{c}0,25( \pm \\
0,5)\end{array}$ & 0 & $132( \pm 113,46)$ & $175( \pm 102,62)$ & 0 & 0 \\
\hline Oligochaeta & $\begin{array}{c}1,75( \pm \\
2,87)\end{array}$ & $2,75( \pm 4,86)$ & 6000 & 4950 & $6,25( \pm 8,02)$ & $0,5( \pm 0,58)$ \\
\hline Leptophlebiidae & 0 & $0,25( \pm 0,5)$ & 0 & 0 & 0 & $1( \pm 0,82)$ \\
\hline Gripopterygidae & $\begin{array}{c}1,5( \pm \\
1,29)\end{array}$ & $6,75( \pm 4,27)$ & 0 & 0 & $12,25( \pm 5,62)$ & $8,75( \pm 3,1)$ \\
\hline Acarii & $2,25(3,86)$ & $1,25( \pm 0,96)$ & 0 & $0,5( \pm 1)$ & $1,5(1,29)$ & $0,75( \pm 1,5)$ \\
\hline Hyalella sp. & $1( \pm 0,82)$ & $0,25( \pm 0,5)$ & 0 & $1( \pm 0,82)$ & $1( \pm 2)$ & $0,25( \pm 0,5)$ \\
\hline Hydridae & 0 & 0 & $36,5( \pm 33,91)$ & $31,75( \pm 29,85)$ & 0 & 0 \\
\hline Nematodes & 0 & 0 & $12,25( \pm 10,7)$ & $2,5( \pm 4,36)$ & 0 & 0 \\
\hline Corixidae & 0 & 0 & 0 & 0 & $0,25( \pm 0,5)$ & 0 \\
\hline Coleoptera & 0 & 0 & 0 & 0,25 & 0 & 0 \\
\hline
\end{tabular}



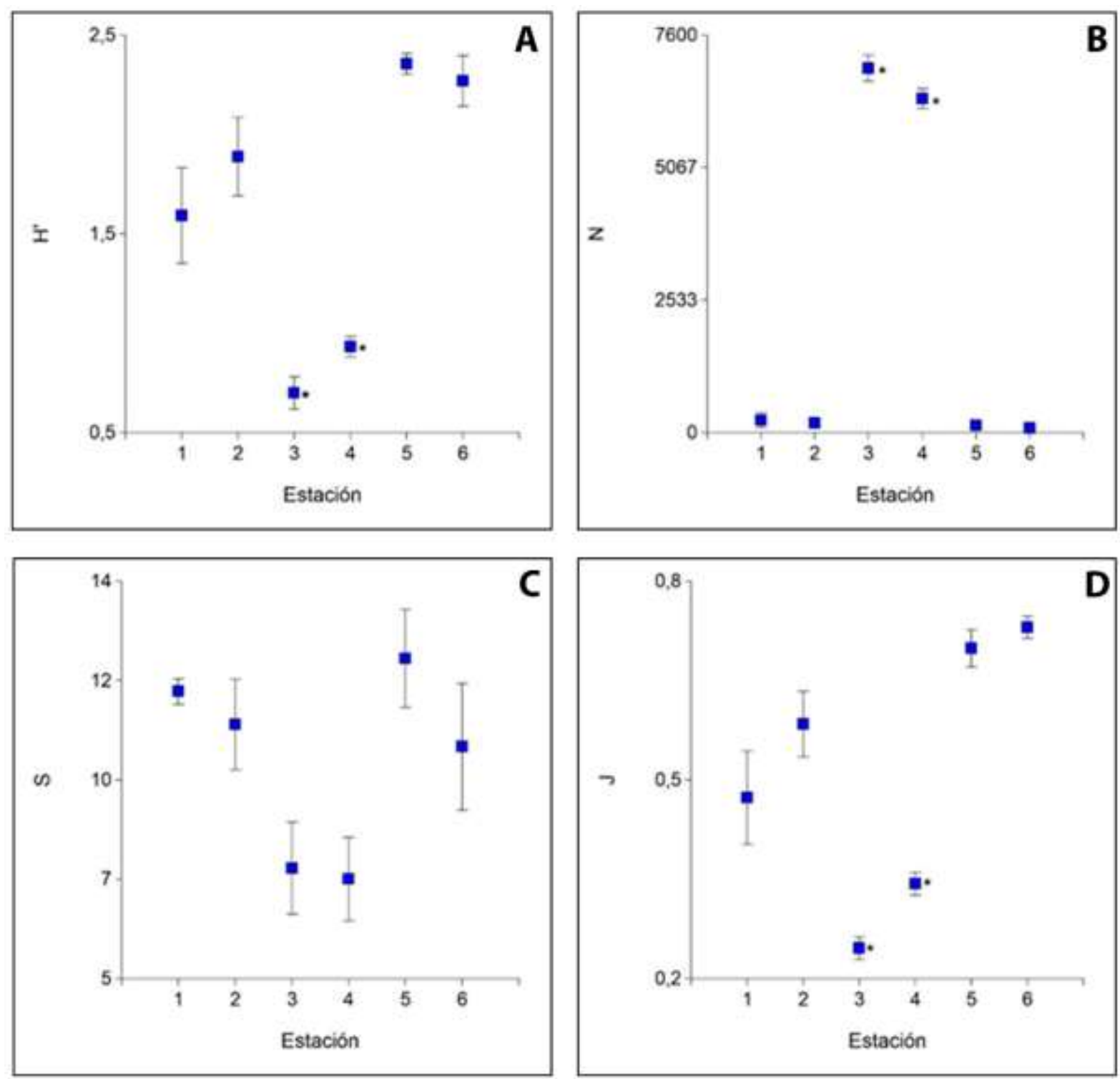

Figura 2. (A) Diversidad (H'), (B) Abundancia total (N), (C) Riqueza de especies (S), (D) Equitatividad $(\mathrm{J})$ de la comunidad en las distintas estaciones. $*$ valores significativamente diferentes (Mann-Whitney $\mathrm{p}<0,05$ ).

Los análisis estadísticos multivariados de agrupamiento y de ordenamiento permitieron identificar dos grupos principales de muestras (ANOSIM: $\mathrm{R}_{\text {global }}=0,86, \mathrm{p}<0,01$; SIMPROF: $\mathrm{Pi}=12,65, \mathrm{p}<0,01$ ) (Fig. 3). El Grupo 1, integrado por las muestras de las estaciones 1, 2, 5 y 6 se encontró caracterizado por Baetidae, Chilina sp. y Gripopterigydae. El Grupo 2, integrado por las muestras de las estaciones 3 y 4, se caracterizó por la dominancia de oligoquetos, quironómidos e hirudíneos del género Helobdella (Tabla 3). Los oligoquetos, los quironómidos y los hirudíneos Helobdella sp. contribuyeron con más del $75 \%$ a la disimilitud entre ambos grupos de muestras (Tabla 4). 


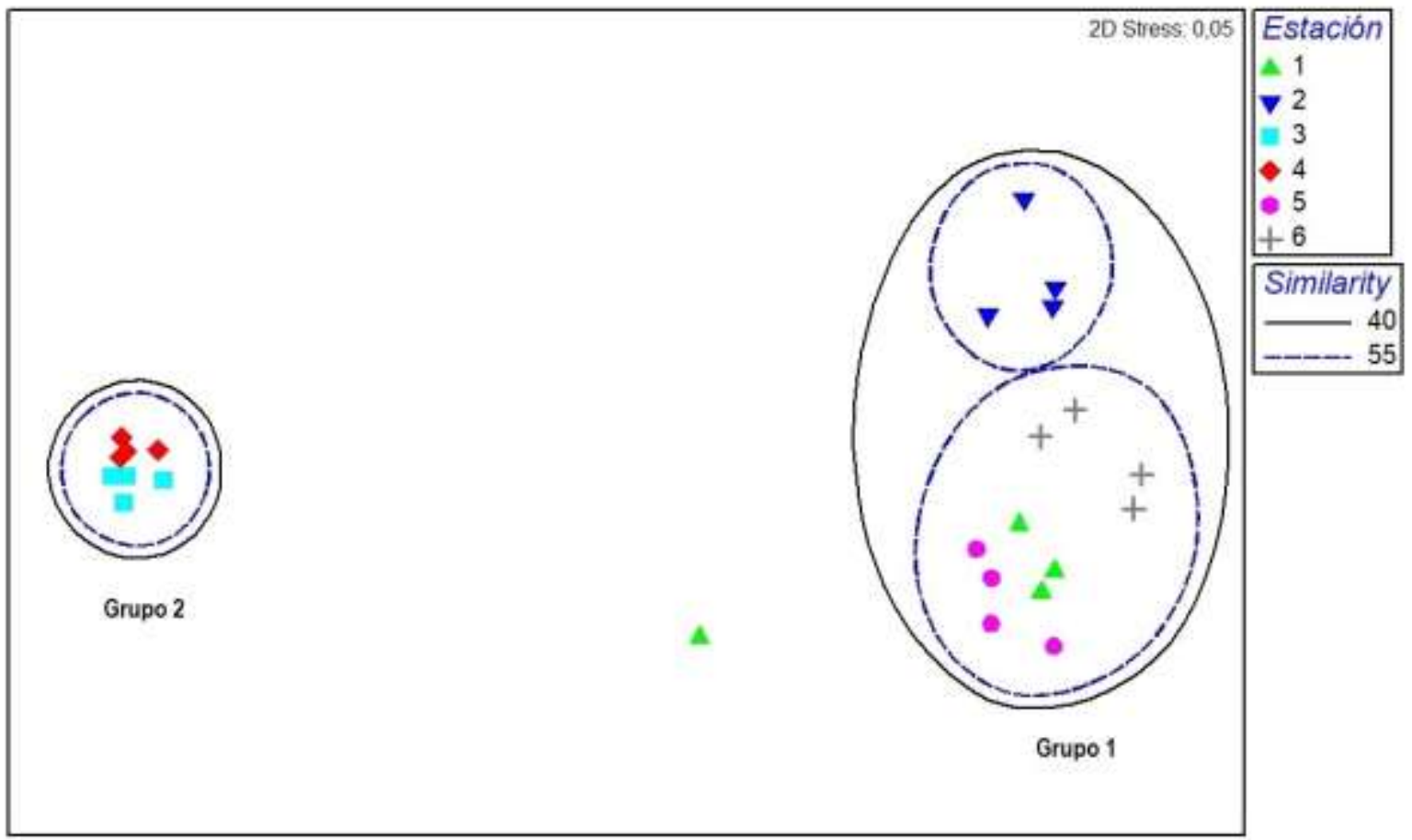

Figura 3. Análisis de ordenamiento (MDS) y agrupamiento de muestras (HAC), utilizando ttransformación raíz cuadrada de la abundancia, índice de similitud de Bray-Curtis y ligamiento promedio.

Tabla 3. Análisis SIMPER*. Taxones que más contribuyeron a la similitud entre muestras en cada grupo.

\section{Ab transf. $\quad \%$ cont. $\%$ acum.}

Grupo 1: Est. 1, 2, 5 y 6

Similitud promedio: 54,95

Baetidae

5,35

27,84

27,84

Chilinidae

4,92

13,62

41,47

Gripopterygidae

2,47

11,36

52,82

Chironomidae

2,65

7,73

60,55

Elmidae

2,16

7,14

67,69

Simulidae

1,57

6,89

74,58

Hydropsychidae

1,38

5,26

79,84

Lymnaeidae

2,85

3,86

83,69

Hydrobiosidae

1,05

3,81

87,50

Glossosomatidae

1,48

3,10

90,60

Grupo 2: Est. 3 y 4

Similitud promedio: 88,94

Oligochaeta

$\begin{array}{ccc}73,91 & 62,94 & 62,94 \\ 31,24 & 25,08 & 88,03 \\ 11,38 & 7,11 & 95,14\end{array}$

Chironomidae

Helobdella $\mathrm{sp}$.
$*$ Ab. transf., abundancia transformada $(\sqrt{ })$ promedio; $\%$ cont., porcentaje de

Helobdella $\mathrm{sp}$.
$*$ Ab. transf., abundancia transformada $(\sqrt{ })$ promedio; $\%$ cont., porcentaje de

95,14 contribución a la similitud; \% acum., porcentaje acumulado de contribución a la similitud. 
Tabla 4. Análisis SIMPER*. Taxones que más contribuyeron a la disimilitud entre grupos de muestras.

\begin{tabular}{lcccc}
\hline & \multicolumn{2}{c}{ Ab. Transf } & \% cont. & \% acum. \\
\hline Dis. prom.: 94,17 & Grupo 1 & Grupo 2 & & \\
\hline Oligochaeta & 1,10 & 73,91 & 48,86 & 48,86 \\
Chironomidae & 2,65 & 31,24 & 19,18 & 68,04 \\
Helobdella sp & 0,06 & 11,38 & 7,38 & 75,41 \\
Hydra sp. & 0 & 5,25 & 3,42 & 78,84 \\
Baetidae & 5,35 & 0,35 & 3,37 & 82,21 \\
Chilinidae & 4,92 & 0,18 & 3,22 & 85,42 \\
Lymnaeidae & 2,85 & 0,3 & 1,91 & 87,34 \\
Gripopterygidae & 2,47 & 0 & 1,67 & 98,01 \\
Elmidae & 2,16 & 0 & 1,43 & 90,43 \\
\hline
\end{tabular}

* Ab. transf., abundancia transformada $(\sqrt{ })$ promedio; \% cont., porcentaje de contribución a la disimilitud; \% acum., porcentaje acumulado de contribución a la disimilitud

La tabla 5 muestra los valores promedio del índice BMPS para las estaciones de muestreo. Las estaciones 3 y 4 presentaron los valores más bajos de BMPS, indicando la existencia de aguas contaminadas a fuertemente contaminadas, mientras que en las estaciones control $(1 \mathrm{y}$ 2) y las más alejadas de la localidad, el índice BMPS indicó mejores condiciones en la calidad ambiental del río. El análisis estadístico mostró diferencias significativas en el valor de BMPS entre las estaciones de muestreo $(\mathrm{H}=16,18, \mathrm{p}=0,006)$. El análisis post-hoc mostró diferencias significativas entre las estaciones 3 y 4 y las estaciones control y las más alejadas río abajo, pero no se observaron diferencias significativas entre las estaciones 1 y 2 y las estaciones 5 y 6 (Tabla 5).

Tabla 5. Valores promedio del BMPS ( \pm d.s.)

\begin{tabular}{cl}
\cline { 2 - 2 } Estación & \multicolumn{1}{c}{ BMPS } \\
\cline { 1 - 2 } 1 & $56,25^{\mathrm{A}}( \pm 7,32)$ \\
2 & $53,25^{\mathrm{A}}( \pm 17,33)$ \\
3 & $14,5^{\mathrm{B}}( \pm 8,14)$ \\
4 & $16^{\mathrm{B}} \quad( \pm 6,16)$ \\
5 & $63,25^{\mathrm{A}}( \pm 6,13)$ \\
6 & $61,25^{\mathrm{A}}( \pm 14,36)$ \\
A, B Valores significativamente diferentes (Mann-Whitney $\mathrm{p}<0,05)$
\end{tabular}

\section{DISCUSIÓN}

Los cambios espaciales observados en la comunidad bentónica en este estudio pueden ser relacionados con las diferentes condiciones ambientales detectadas en las distintas estaciones de muestreo. La elevada abundancia y la amplia dominancia de organismos tolerantes a impactos ambientales, como las especies de oligoquetos mencionadas, los hirudíneos y los quironómidos en las estaciones 3 y 4, muestran la existencia de alteración ambiental, que se ve reflejada también en el aumento de los valores de $\mathrm{P}$ total, de $\mathrm{N}-\mathrm{NH}_{4}$ y de materia orgánica particulada en el agua. Las causas de la alteración en la calidad del agua del río pueden ser 
variadas, pudiendo tener su origen en el aporte de fuentes difusas, como la actividad agrícola ganadera que se desarrolla en la zona de estudio, y en fuentes puntuales, como el vertido del efluente proveniente de la planta de tratamiento cloacal de la localidad.

El agrupamiento y el ordenamiento de las muestras, resultante del análisis multivariado, demuestra una clara relación espacial entre la estructura de la comunidad y la ubicación de las diferentes estaciones de muestreo con respecto a las principales fuentes de impacto. Los taxones que caracterizaron a las muestras del Grupo 1 son los que, en general, se relacionan a condiciones ambientales más favorables, como los órdenes de insectos Ephemeroptera, Plecoptera y Trichoptera (taxones sensibles), mientras que los taxones considerados tolerantes caracterizaron a las muestras del Grupo 2 y fueron los que más contribuyeron al agrupamiento de las muestras de las estaciones 3 y 4.

Las estaciones 3 y 4 (más cercanas aguas abajo a la localidad de Gobernador Gregores), presentaron los valores promedio más bajos del índice Biotic Monitoring Patagonian Streams (BMPS), ubicándose dentro de las categorías de calidad de agua $\mathrm{V}$ (aguas fuertemente contaminadas) y IV (aguas contaminadas) respectivamente. Las estaciones 1 y 2 (control) y las estaciones 5 y 6 (más alejadas río abajo de la localidad) presentaron los valores más altos del índice, ubicándose en las categorías III (aguas probablemente contaminadas) y II (aguas con contaminación incipiente), respectivamente (Fig. 5). Sin embargo, los valores promedio de las estaciones control ( 1 y 2 ) no mostraron diferencias estadísticas significativas con aquellos de las estaciones más alejadas de la localidad (5 y 6), a pesar de que el valor del índice BMPS le otorga a estas estaciones categorías de calidad de agua diferentes, según la escala propuesta por Miserendino y Pizzolón (1999). Esto pone en evidencia la necesidad de ser cautos al interpretar los resultados del índice, debiendo realizarse mayor número de muestreos que incluyan preferentemente diferentes épocas del año, antes de arribar a un diagnóstico definitivo sobre la calidad ambiental de un cuerpo de agua. 


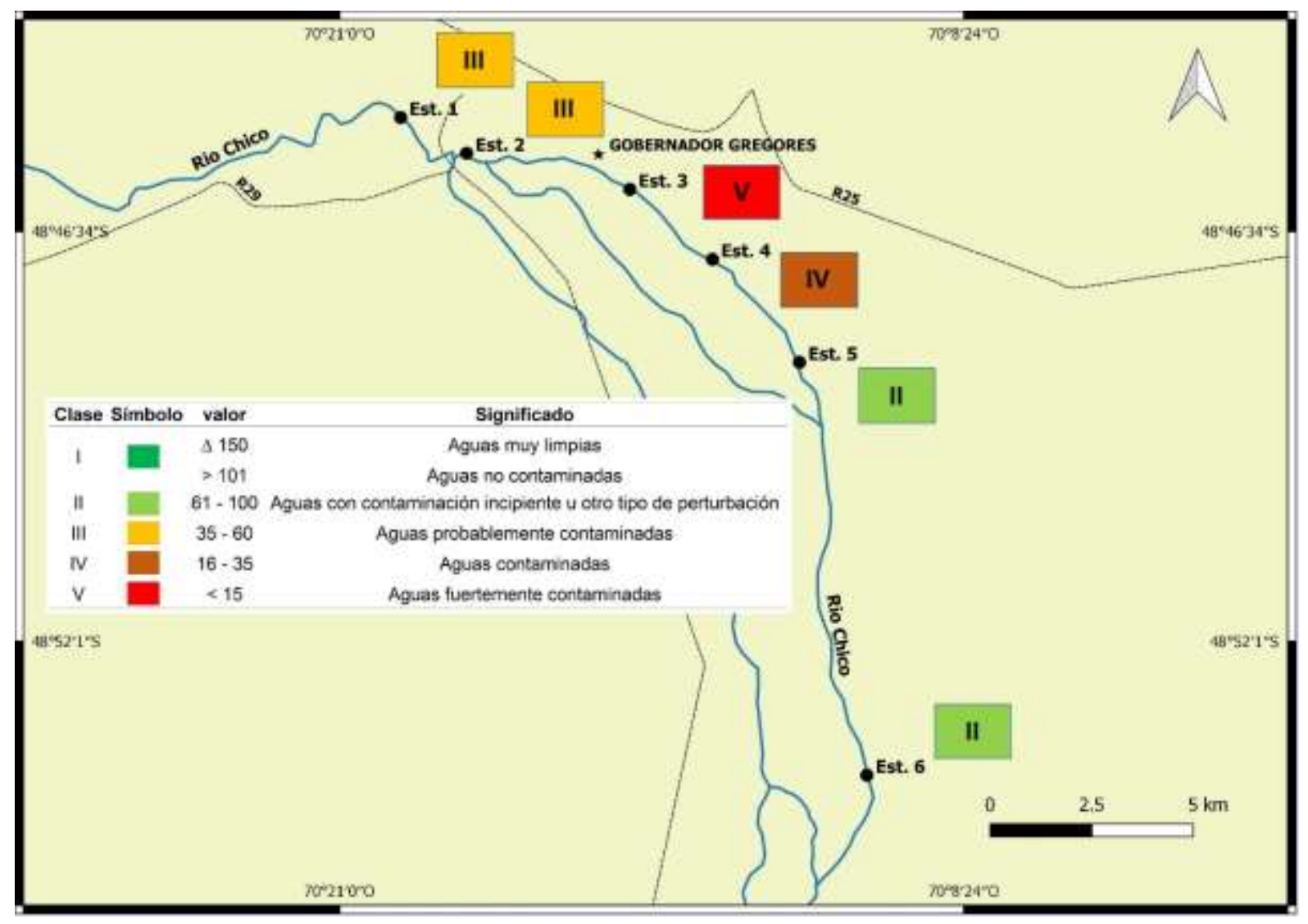

Figura 5. Categorías de calidad de agua asignadas a las estaciones de muestreo según el índice BMPS (modificado de Miserendino y Pizzolón, 1999).

Muchos trabajos científicos han demostrado que la utilización de índices bióticos basados en la comunidad de macroinvertebrados son una herramienta útil para el monitoreo de cuerpos de agua (Miserendino y Pizzolón 1999; Rodrigues Capítulo et al., 2001, Prat et al., 2009; Miserendino y Brand 2009). Asueta (2017), evaluó la aplicación de cuatro índices bióticos en la cuenca superior del río Gallegos, comprobando que el índice BMPS mostró mayor correlación con las variables ambientales vinculadas con la calidad del agua, dando resultados satisfactorios para el monitoreo de una cuenca de la provincia de Santa Cruz.

Por su fácil aplicación y sus bajos costos económicos, el índice BMPS muestra ser una herramienta de diagnóstico adecuada y de gran utilidad para el monitoreo del estado ambiental de los recursos hídricos de la provincia de Santa Cruz. Si bien este índice es aplicable a la zona de estudio y muestra una buena respuesta frente a las problemáticas ambientales de la provincia, debe señalarse que fue diseñado para la biozona andina de la provincia de Chubut, siendo necesaria su adaptación y calibración para aplicarlo de manera más eficiente y confiable en la Patagonia extra-andina de Santa Cruz. Esto implica que deben revisarse los puntajes de sensibilidad/tolerancia otorgado originalmente a algunas familias de invertebrados, requiriéndose también la inclusión de otros taxones que son importantes en términos de presencia y abundancia en la comunidad macrobentónica de la provincia de Santa Cruz. 


\section{REFERENCIAS BIBLIOGRAFICAS}

ARMENDÁRIZ LC., CORTESE B., RODRÍGUEZ M., RODRIGUES CAPITULO A. 2017. Ecosystem services of runoff marshes in urban lowland basins: Proposals for their management and conservation. Knowledge and Management of Aquatic Ecosystems 112. https://doi.org/10.1051/kmae/2017022

ASSEF, Y., HORAK, C., BOQUET, M. 2014. Characterization of the multixenobiotic resistance (MXR) mechanism in the freshwater snail Physa acuta from Patagonia (Argentina). New Zealand Journal of Marine and Freshwater Research. vol. 48 p. 86 - 96 https://doi.org/10.1080/00288330.2013.846921

ASUETA, R., SÚNICO, A. MARTIN, J. P. 2011. Análisis y caracterización de macroinvertebrados bentónicos en la cuenca superior del río Gallegos - Santa Cruz. Su aplicación para la evaluación de la calidad ambiental de un recurso hídrico afectado por la extracción y uso del carbón para la generación de energía. 2do Encuentro de Investigadores de la Patagonia Austral. Universidad Nacional de la Patagonia Austral. https://doi.org/10.4995/thesis/10251/2626

ASUETA, R. 2017. Análisis y caracterización de macroinvertebrados bentónicos en la cuenca superior del río Gallegos - Santa Cruz. Su aplicación para la evaluación de la calidad ambiental de un recurso hídrico afectado por la extracción y uso del carbón para la generación de energía. Tesis de Maestría en Manejo y Gestión de los Recursos Naturales en la Patagonia, Universidad Nacional de la Patagonia Austral. https://doi.org/10.4995/thesis/10251/4771

CLARKE K. R. Y WARWICK R. M. 2001. Change in marine communities: an approach to statistical analysis and interpretation. PRIMER-E, Plymouth, $159 \mathrm{pp}$.

GODOY MANRIQUEZ, C. J. (Dir.). 2000. El gran libro de la Provincia de Santa Cruz. Milenio Ediciones y Alfa Centro Literario.

GRAY N. F. Y DELANEY E. 2008. Comparison of benthic macroinvertebrate indices for the assessment of the impact of acid mine drainage on an Irish river below an abandoned $\mathrm{Cu}-\mathrm{S}$ mine. Environmental. Pollution. 155:31-40. https://doi.org/10.1016/j.envpol.2007.11.002

HORAK C., ASSEF Y. 2017. Influence of Water Temperature on the MXR Activity and Pglycoprotein Expression in the Freshwater Snail, Physa acuta (Draparnaud, 1805). Zoological studies. 56 (56): 1-11.

MARTIN J. P., SAR A., FERNÁNDEZ R., CAMINOS C., PEREYRA GINESTAR B., MOSCARDI C., GASPARI G. 2014. Diversidad de macroinvertebrados bentónicos y aplicación de índices bióticos de calidad ambiental en el Río Santa Cruz (Patagonia austral, Argentina). Memorias del II Congreso Internacional de Hidrología de Llanuras. https://doi.org/10.30972/fac.310650

MASI, C. Y MISERENDINO, M. L. 2009. Usos de la tierra y distribución de la materia orgánica particulada béntica en ríos de montaña. Ecología austral 19: 185-196.

MISERENDINO, M. L. 2004. Effects of landscape and desertification on the macroinvertebrate assemblages of rivers in Andean Patagonia Archiv fur hydrobiologie 159 (2):185-209. https://doi.org/10.1127/0003-9136/2004/0159-0185

MISERENDINO M. L. Y BRAND C. 2009. Environmental effects of urbanization on streams and rivers in Patagonia (Argentina): the use of macroinvertebrates. In: Daniels J.A. (ed.). Monitoring Advances in Environmental Research, Volume 6, Nova Science Publishers, Inc.: 1-38.

MISERENDINO, M. L. Y PIZZOLÓN, L. 1999. Rapid assessment of river water quality using macroinvertebratess: a family level biotic index for the Patagonic Andean zone. Acta Limnologica Brasiliensia 11:137-148. 
PRAT N., RÍOS B., ACOSTA R., RIERADEVALL M. 2009. Los macroinvertebrados como indicadores de calidad de las aguas. En: Domínguez E. y Fernández H (ed.). Macroinvertebrados bentónicos sudamericanos: Sistemática y biología. - 1a ed. Tucumán: Fundación Miguel Lillo, pp. 631 - 654. https://doi.org/10.1603/008.103.0401

RED HIDROLÓGICA NACIONAL. 2013. Publicaciones Hidrometeorológicas 2012. Subsecretaria de Recursos Hidricos, Ministerio de Planificación Federal, Inversión Pública y Servicios

RIVERA VELASCO S., TORRES S., MARTIN JP., TELLO G. 2017. Caracterización de la comunidad de macroinvertebrados bentónicos en un sector del sudoeste de Santa Cruz, Patagonia Austral, Argentina. Libro de trabajos completos, III Congreso Nacional de Ciencia y Tecnología Ambiental Pp:73- 78. https://doi.org/10.24850/j-tyca-2017-06-05

RODRIGUES CAPÍTULO, A., TANGORRA M, OCÓN C. 2001. Use of benthic macroinvertebrates to assess the biological status of Pampean streams in Argentina. Aquat. Ecol. 35 (2): 109-119.

ROSENBERG, D. M. Y RESH, V. H. (1993). Freshwater Biomonitoring and Benthic Invertebrates. Chapman \& Hall: New York.

ROY, A. H., ROSEMOND, A. D., PAUL, M. J., LEIGTH, D. S., WALlACE, B. (2003). Stream macroinvertebrate response to catchment urbanization (Georgia, USA). Freshwater Biology, 48, 329-346. https://doi.org/10.1046/j.1365-2427.2003.00979.x

SUREN, A. M. (2000). Effects of urbanization. In: K.J. Collier, M.J. Winterbourn (Eds.), New Zealand invertebrates: ecology and implications for management (pp. 260-268) Christchurch, New Zealand: New Zealand Limnological Society.

TAGLIAFERRO M., MISERENDINO L., LIBEROFF AL, QUIROGA P., PASCUAL M. 2013. Dams in the Last Large Free-Flowing Rivers of Patagonia, the Santa Cruz River, Environmental Features, and Macroinvertebrate Community. Limnologica 43: 500 509. https://doi.org/10.1016/j.limno.2013.04.002

TAGLIAFERRO M., QUIROGA, A., PASCUAL M. A. 2014. Spatial Pattern and Habitat Requirements of Galaxias maculatus in the Last Un-Interrupted Large River of Patagonia: A Baseline for Management. Environment and Natural Resources Research 4 (1): 54-63. https://doi.org/10.5539/enrr.v4n1p54

WEATHERLEY N. S. Y ORMEROD S. J. 1992. The utility of biological indicators of streams acidity in Wales. En Mckenzie D. H., Hyatt D. E., Mcdonald, V. J. (ed.) 1992. Ecological Indicators. Vol 2. Elsevier Applied Science London and New york. Cap. 76. Pp 1341-1354. https://doi.org/10.1007/978-1-4615-4661-0_37 